\section{Biomarcadores para el diagnóstico de la Enfermedad de Alzheimer}

Manuel Menéndez González, Ciara García, Esther Suárez-Sanmartín, Santiago Fernández, Rocío Álvarez-Escudero, Marta Blázquez

\section{Resumen}

La Enfermedad de Alzheimer se diagnostica en base a criterios clínicos y su diagnóstico precoz, en fases prodrómicas, continúa siendo un reto. Las pruebas complementarias que se realizan rutinariamente ante un caso de sospecha no van dirigidas a evidenciar la presencia de la enfermedad, sino a descartar otros procesos que pueden producir un cuadro clínico similar. Estudios prospectivos han demostrado que los diagnósticos de Enfermedad de Alzheimer eran erróneos en aproximadamente la cuarta parte de los casos, aún en centros especializados. La solución a este reto son los biomarcadores; pruebas que permitan confirmar o excluir la presencia de la enfermedad.

En este artículo se describen sucintamente los cuatro tipos de biomarcadores aceptados para apoyar el diagnóstico de Enfermedad de Alzheimer: las pruebas de laboratorio, la neuroimagen estructural, la neuroimagen funcional y la neuroimagen molecular. También se discuten someramente los aspectos más relevantes de su utilización en la práctica clínica diaria.

Palabras clave: Alzheimer, deterioro cognitivo leve, biomarcadores, PET, RM, tau, amiloide
Servicio de Neurología, Hospital Universitario Central de Asturias.

\section{Correspondencia:}

$\equiv$ manuelmenendez@gmail.com

\section{Introducción}

De forma muy resumida, y de acuerdo a la teoría de la cascada amiloide [1], la enfermedad de Alzheimer (EA) se produce debido a que en la membrana neuronal se generan unos péptidos de amiloide de 42 aminoácidos (beta-amiloide), que resultan insolubles (en contraposición al péptido amiloide fisiológico, de 40 aminoácidos, que es soluble). Los monómeros de beta-amiloide se van agregando en agrupaciones cada vez más complejas hasta formar placas extracelulares de amiloide. Paralelamente, en el interior de las neuronas, una proteína encargada de estabilizar los neurotúbulos, denominada proteína Tau, es fosforilada de un modo excesivo, con lo que se altera la integridad de los neurotúbulos neuronales y producen unos agregados intraneuronales denominados ovillos neurofibrilares (Figura 1).

La razón última por la que se inicia este proceso resulta desconocida, salvo en aquellos casos (los menos), en los que la enfermedad tiene un origen genético. A pesar de las lagunas en el conocimien- 


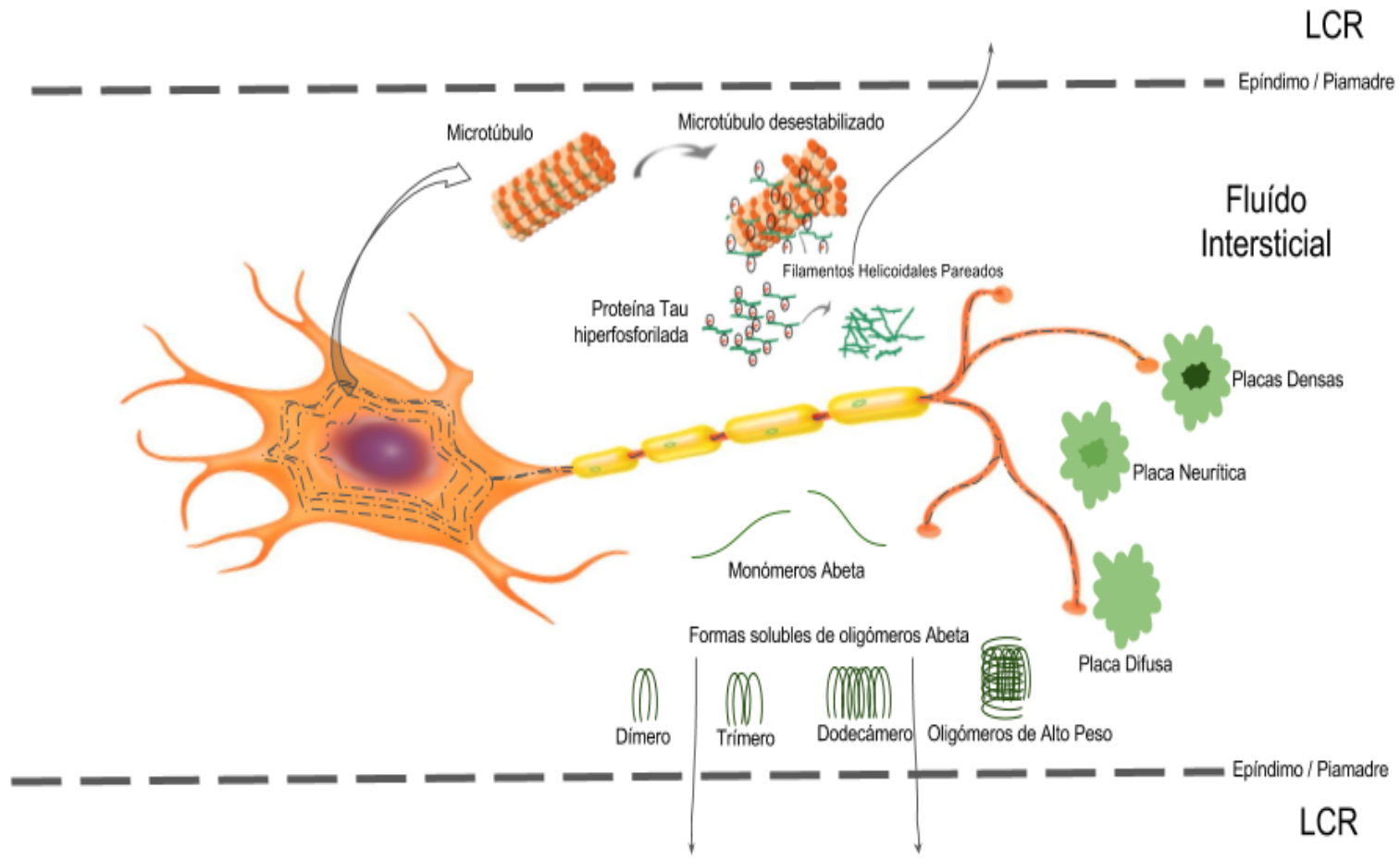

Figura 1. Neuropatología de la tntermedad de Alzheımer. tn la parte superıor se representa la hipertostorılación ıntraneuronal de la proteína Tau que termina por desestabilizar los microtúbulos. En la parte inferior se representan las formas solubles de Abeta y su depósito en placas amiloides extracelulares. Tanto las formas solubles de Abeta como la proteína Tau, difunden desde el FIS hasta el LCR, donde pueden ser cuantificadas.

to de la fisiopatología de la enfermedad, hay dos hechos que sí son bien conocidos y universalmente aceptados, como son: que el proceso neurodegenerativo se inicia años (décadas) antes de la aparición de la clínica; y que este proceso neurodegenerativo no se inicia en todas las regiones de la corteza cerebral al mismo tiempo, sino que existe una vulnerabilidad selectiva de ciertas regiones. De este modo, la progresión de la enfermedad sigue una distribución característica, iniciándose en corteza del hipocampo y amígdala cerebral, desde donde progresa a la circunvolución parahipocampal, y posteriormente a otras regiones de la corteza de los lóbulos temporales y frontal y sistema límbico.

Desde su descripción, la EA se diagnostica en base a criterios clínicos [3-5]. Las pruebas complementarias que se realizan de forma rutinaria ante un caso de sospecha no van dirigidas a evidenciar la presencia de la enfermedad, sino únicamente a descartar otros procesos que pueden producir un cuadro clínico similar. Así, por ejemplo, a todos los sujetos con deterioro cognitivo se les solicitaba una analítica con niveles de vitamina B12 y ácido fólico - para excluir deterioro cognitivo carencial-, TSH - para excluir hipotiroidismo-, serologías - para descartar enfermedades infecciosas del SNC- y una prueba de imagen cerebral, generalmente un TC craneal -para descartar hidrocefalia normotensiva, lesiones vasculares o lesiones ocupantes de espacio-. Estudios prospectivos demostraron que los diagnósticos de EA eran erróneos en el 20\%-30\% de los casos, aún en centros especializados [6]. Esta dificultad es más relevante en las fases más iniciales de la enfermedad, cuando la clínica es más sutil.

En los últimos 20 años se ha realizado un importante esfuerzo investigador para poder contar 
con pruebas que permitan detectar la presencia de la enfermedad de Alzheimer; a estas pruebas las denominados biomarcadores de Alzheimer. Hoy en día contamos con cuatro tipos de biomarcadores aceptados para apoyar el diagnóstico de EA: las pruebas de laboratorio, la neuroimagen estructural, la neuroimagen funcional y la neuroimagen molecular [7].

\section{Descripción de los principales biomarcadores para el diagnóstico precoz de la Enfermedad de Alzheimer}

\section{Pruebas de laboratorio}

Las formas solubles de proteína beta-amiloide y proteína tau difunden desde el Fluído Intersticial (FIS) al Líquido Cefalorraquídeo (LCR) donde pueden ser cuantificadas [2]. Se ha demostrado que tanto la disminución de la concentración de proteína beta-amiloide, como la elevación de proteína tau, especialmente en su forma hiperfosforilada, son marcadores sensibles y específicos de la EA, incluso desde fases muy iniciales de la misma (sujetos presintomáticos). Tanto es así, que ya han dado el salto desde la investigación a la clínica, y desde hace unos años se utilizan como ayuda diagnóstica en aquellos casos en los que se sospecha la enfermedad pero existen dudas al respecto. Sin embargo, a pesar de su evidente utilidad, también presentan algunos inconvenientes. Los principales están relacionados con la necesidad de realizar una punción lumbar, y que los resultados varían notablemente de unos laboratorios a otros, lo que dificulta la interpretación de los mismos. Existe, por tanto, una importante necesidad de contar con pruebas de laboratorio que puedan realizarse sobre muestras periféricas (plasma, orina o saliva) y que los resultados sean reproducibles.

\section{Neuroimagen estructural}

La topografía típica de la enfermedad, que produce un patrón de atrofia característico (iniciándose en el hipocampo y circunvolución parahipocampal) puede ser visualizable mediante resonancia magnética (Figura 2). Particularmente, la volumetría del hipocampo ha demostrado ser una técnica sensible y relativamente específica de la enfermedad, además de ser no invasiva y reproducible. Sin embargo estos cambios no son tan precoces como los bioquímicos, por lo que no es útil en fases presintomáticas. Además, otras enfermedades neurodegenerativas también pueden cursar con atrofia del hipocampo, por lo que no es una medida muy específica. También presenta el inconveniente de precisar métodos de cuantificación que requieren conocimientos radiológicos específicos y considerable tiempo para su ejecución, por lo que hoy en día aún no se suelen realizar de forma sistemática en la práctica clínica.

\section{Neuroimagen funcional}

El PET de glucosa (FDG-PET) mide el metabolismo cerebral. En la EA se produce un patrón de metabólico característico, con hipometabolismo en regiones posteriores del lóbulo parietal, precuneus, y regiones de la corteza frontal, respetando la corteza sensitivo-motora y visual. Numerosos estudios

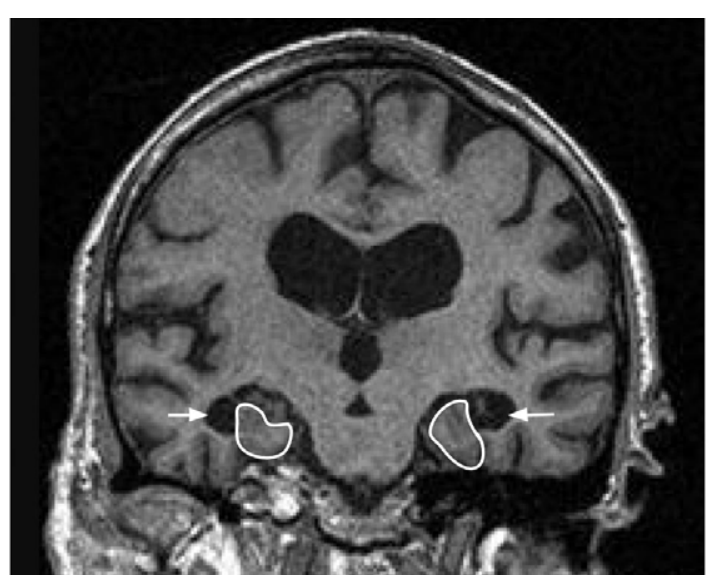

Figura 2. Paciente con Enfermedad de Alzheimer de 68 años de edad, en el que se aprecia atrofia cortical difusa pero especialmente pronunciada a nivel de ambos hipocampos y circunvoluciones parahipocampales (áreas contorneadas) y dilatación secundaria de las astas temporales de los ventrículos laterales (flechas). 

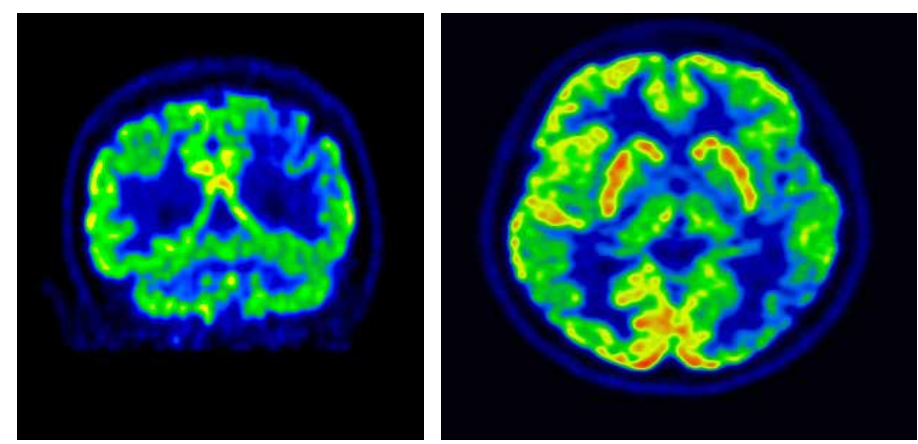

Figura 3. FDG-PET de paciente de 55 años con deterioro cognitivo leve debido a Enfermedad de Alzheimer. A la izquierda corte axial, a la derecha corte coronal. Se observa una marcada disminución de la actividad de la corteza de asociación posterior del cerebro de forma bilateral, que afecta fundamentalmente a la corteza parietal, incluida la corteza angular, y parcialmente el cíngulo posterior, con claro predominio hemisférico izquierdo. Igualmente, la actividad de la corteza temporal lateral y medial izquierda se encuentra disminuida. El metabolismo de la corteza primaria tanto motora, como auditiva y visual, se encuentra conservado.

han demostrado que estas alteraciones están presentes desde fases muy iniciales de la enfermedad, particularmente el hipometabolismo en el cíngulo posterior y precuneus. En pacientes con deterioro cognitivo leve tiene un alto valor predictivo, tanto positivo como negativo (Figura 3).

\section{Neuroimagen molecular}

Los PET que utilizan como isótopos radioligandos que se unen a proteínas permiten valorar la carga de estas proteínas en el tejido cerebral. De este modo, los PET de amiloide y de tau nos permiten conocer en vivo si el cerebro de un paciente presenta depósito de una amiloide y/o depósito de tau. Además, cada enfermedad tiene una distribución característica de los depósitos. Así, en la EA, se describe un depósito de amiloide en la corteza cerebral, incluyendo regiones frontales y temporoparietales y precuneus. La validez de los hallazgos de PET-amiloide y PET-Tau ha sido confirmada por estudios neuropatológicos postmortem. El PET de amiloide tiene un alto valor pronóstico negativo, de modo que un resultado negativo prácticamen- te excluye la enfermedad. Sin embargo, un PET de amiloide positivo, por sí sólo, no es sinónimo de padecer la enfermedad.

\section{Utilización clínica de los biomarcadores}

Los nuevos criterios para el diagnóstico de la EA ya incluyen los 4 tipos de biomarcadores como criterios "de apoyo" para el diagnóstico de la enfermedad [5]. Sin embargo, hay que señalar que estos marcadores presentan diferencias en su significación clínica [7-9]. Por ejemplo es importante conocer que existe una secuencia cronológica en la aparición de los hallazgos descritos, lo que afecta su interpretación clínica (Figura 4). Las primeras alteraciones en tener lugar son las relacionadas con el beta-amiloide. Así, la disminución de beta-amiloide en LCR y el PET de amiloide, son los biomarcadores que se alteran más precozmente. Tiempo más tarde se produce el hipometabolismo regional evidenciable mediante FDG-PET. Tiempo después se describen los cambios en tau, evidenciables mediante LCR y PET-Tau. Finalmente se producen los cambios estructurales visibles en resonancia. Igualmente, la correlación entre la intensidad de la alteración de los distintos marcadores, con grado de progresión clínica, sólo se comporta de modo lineal con las pruebas de neuroimagen estructural y neuroimagen metabólica, no así con las pruebas de laboratorio y neuroimagen molecular [7].

Además, existen otros aspectos que cabe considerar de cara a su uso en la práctica clínica diaria, como su coste, puesta a punto de la técnica, listas de espera en cada centro, etc...

En los próximos años continuaremos viendo avances en el desarrollo de nuevas pruebas que permitan mejorar aún más el diagnóstico en las fases iniciales de la enfermedad. Incluso se podría llegar a detectar el inicio de la enfermedad en sujetos asin- 


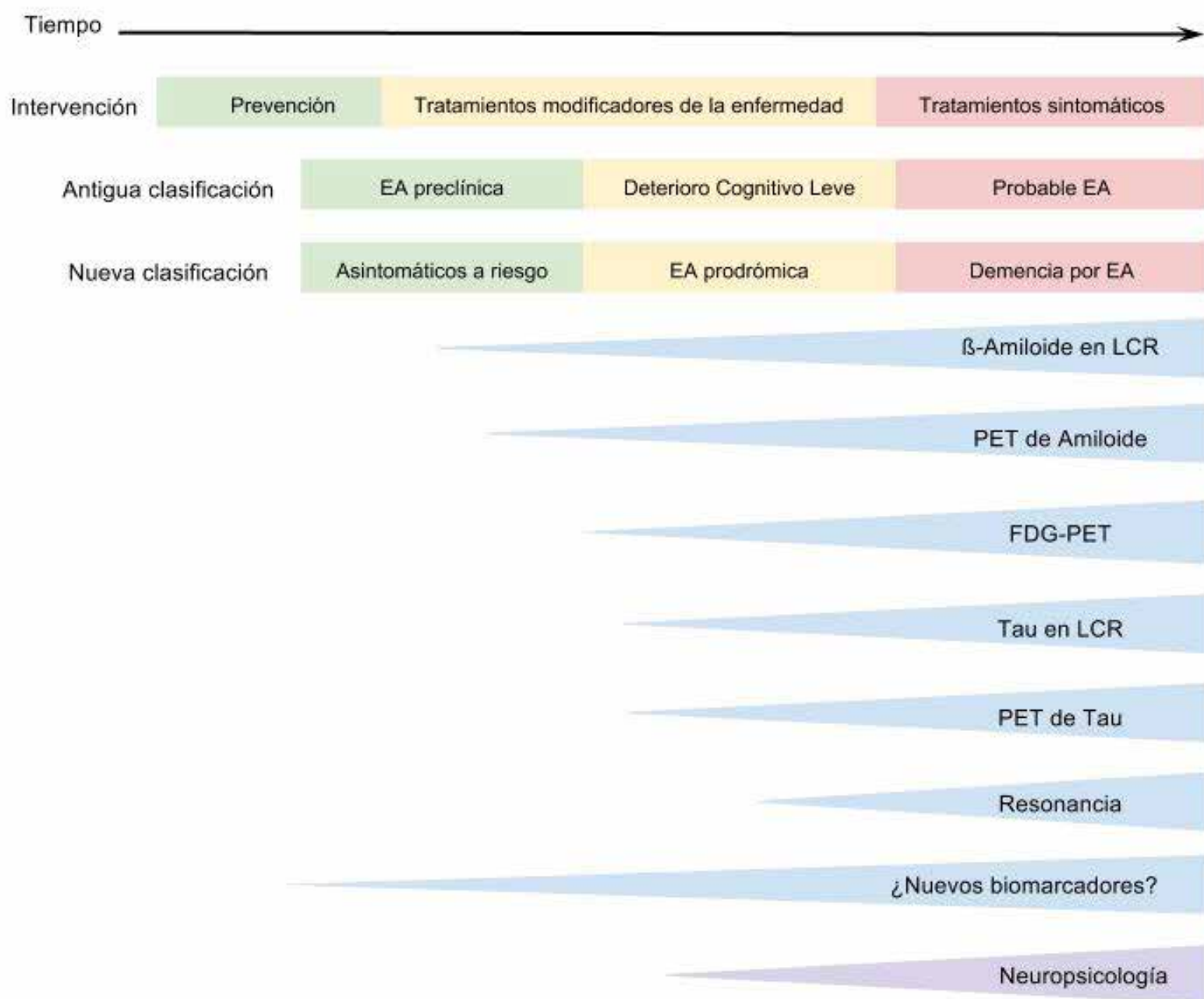

Figura 4. Evolución de la Enfermedad de Alzheimer con la terminología antigua, basada en el concepto de deterioro cognitivo leve y Enfermedad de Alzheimer preclínica; y la nueva terminología basada en los conceptos de EA prodrómica y sujetos asintomáticos a riesgo. Este cambio de paradigma es facilitado por la disponibilidad de biomarcadores, que pueden detectar la presencia de la enfermedad desde fases precoces. A su vez, el diagnóstico precoz permitirá mejores estrategias preventivas y terapéuticas.

tomáticos. Esto tendría implicaciones éticas; pero también ofrecería la oportunidad de realizar intervenciones antes de que el daño neuronal se haya establecido, ya que permitiría diseñar mejores estrategias para abordar la prevención de la enfermedad, identificando aquellos sujetos con riesgo especial, y tratamientos neuroprotectores y modificadores de la enfermedad antes de que el daño neuronal se haya establecido (Figura 4).

En consecuencia, el diagnóstico de la EA está evolucionando desde una aproximación "por exclusión", en el que ante un caso de sospecha el diagnóstico era asumido en ausencia de otros procesos que justifiquen el cuadro clínico; a una aproxima- ción "por confirmación", en el que ante un caso de sospecha, el diagnóstico es confirmado o refutado en base a biomarcadores.

\section{Conclusión}

En las últimas décadas se han hecho avances notables en las pruebas de apoyo para el diagnóstico de la EA. Algunos de estos avances ya han pasado de la investigación a la práctica clínica, de modo que hoy se puede realizar el diagnóstico de la enfermedad de un modo más precoz y más seguro. Todo 
ello redunda en mejores estrategias para abordar la prevención y el tratamiento de la enfermedad.

\section{Referencias}

1. Hardy J, Selkoe DJ. The amyloid hypothesis of Alzheimer's disease: progress and problems on the road to therapeutics. Science 2002;297:353-356

2. Tarasoff-Conway JM, Carare RO, Osorio RS, Glodzik L, Butler T, Fieremans E, Axel L, Rusinek H, Nicholson C, Zlokovic BV, Frangione B, Blennow K, Ménard J, Zetterberg $H$, Wisniewski $T$, de Leon MJ. Clearance systems in the brain--implications for Alzheimer disease. Nat Rev Neurol. 2016;12(4):248. doi: 10.1038/nrneurol.2016.36

3. Sperling RA, Aisen PS, Beckett LA, Bennett DA, Craft S, Fagan AM, Iwatsubo T, Jack CR Jr, Kaye J, Montine TJ, Park DC, Reiman EM, Rowe CC, Siemers E, Stern Y, Yaffe K, Carrillo MC, Thies B, Morrison-Bogorad M, Wagster MV, Phelps CH. Toward defining the preclinical stages of Alzheimer's disease: recommendations from the National Institute on Aging-Alzheimer's Association workgroups on diagnostic guidelines for Alzheimer's disease. Alzheimers Dement. 2011;7(3):280-92. Doi: 10.1016/j. jalz.2011.03.003

4. Jack CR Jr, Albert MS, Knopman DS, McKhann GM, Sperling RA, Carrillo $\mathrm{MC}$, Thies $\mathrm{B}$, Phelps $\mathrm{CH}$. Introduction to the recommendations from the National Institute on AgingAlzheimer's Association workgroups on diagnostic guidelines for Alzheimer's disease. Alzheimers Dement. 2011;7(3):257-62. doi:10.1016/j.jalz.2011.03.004

5. Albert MS, DeKosky ST, Dickson D, Dubois B, Feldman HH, Fox NC, Gamst A, Holtzman DM, Jagust WJ, Petersen RC, Snyder PJ, Carrillo MC, Thies B, Phelps CH. The diagnosis of mild cognitive impairment due to Alzheimer's disease: recommendations from the National Institute on Aging-Alzheimer's Association workgroups on diagnostic guidelines for Alzheimer's disease. Alzheimers Dement. 2011;7(3):270-9. doi: 10.1016/j. jalz.2011.03.008

6. Beach TG, Monsell SE, Phillips LE, Kukull W. Accuracy of the clinical diagnosis of Alzheimer disease at National Institute on Aging Alzheimer Disease Centers, 2005-2010. J Neuropathol Exp Neurol. 2012;71(4):266-73. doi:10.1097/ NEN.0b013e31824b211b
7. Menéndez M. Atlas of Biomarkers for Alzheimer's Disease. Springer 2014. doi: 10.1007/978-3-319-07989-9

8. Menéndez González M. Calatayud MT. Atención al paciente con deterioro cognitivo de origen neurodegenerativo. iMedPub 2015 ISBN: 978-1505868432

9. Menéndez González M. Clinical use of biomarkers for neurodegenerative disorders. Frontiers Media 2014 ISBN: 9782-88919-400-1 ISSN 1664-8714 Doi 10.3389/978-2-88919$400-1$

\section{Opina sobre este artículo:}

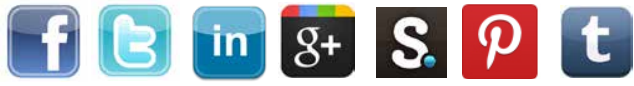

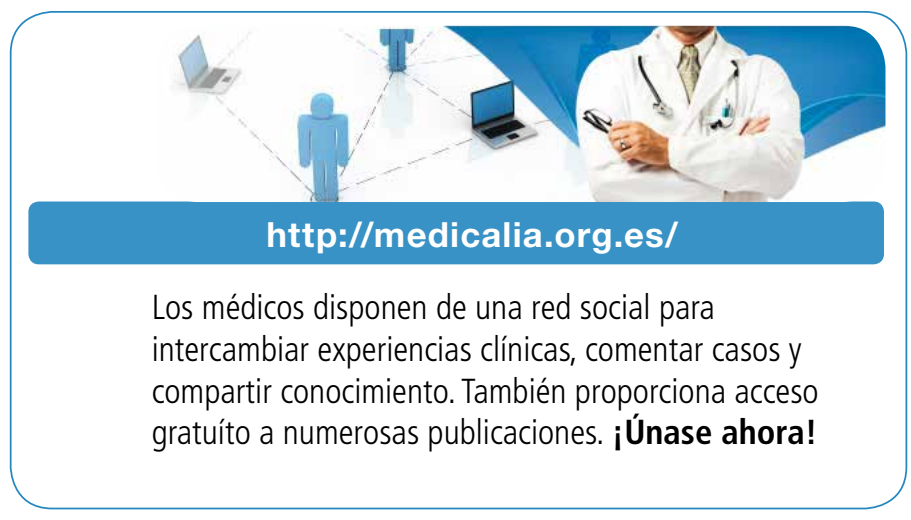

\section{Publique con iMedPub}

http://www.imed.pub

\section{Biomedicina}

$\checkmark$ Biomedicina es una revista de acceso abierto en Español, dedicada a todas las áreas de la medicina, tanto básicas como clínicas.

$\checkmark$ Recoge artículos de investigación originales, revisiones y casos clínicos. 